\title{
Zeitschrift für Erziehungswissenschaft 2010 - Ein Jahresrückblick
}

\author{
Harm Kuper
}

Liebe Leserinnen und Leser der Zeitschrift für Erziehungswissenschaft!

Auf ihrer letzten Sitzung haben die Herausgeberinnen und Herausgeber entschieden, die Jahrgänge der ZfE künftig mit einem Jahresrückblick abzuschließen. Der Rückblick soll das Profil der ZfE im Lichte aktueller Entwicklungen in der Erziehungswissenschaft bilanzieren. Wir erhoffen uns davon eine gesteigerte Aufmerksamkeit auf die im letzten Jahr behandelten Schwerpunkte. Gleichzeitig möchten wir Ihnen die Gelegenheit geben, das thematische Spektrum der ZfE im vergangenen Jahr zu überblicken, und Sie damit zum Einreichen neuer Manuskripte und Vorschläge für innovative Schwerpunkte anregen.

Die vier Themenschwerpunkte der ZfE im Jahr 2010 - Transfer und Transferforschung in der Erziehungswissenschaft; Zivilgesellschaft und freiwilliges Engagement; Gestaltungsspielräume des Lernens Erwachsener; Mehrsprachigkeit - bilden selbstverständlich nur einen Teil der ausgesprochen vielfältigen Forschungsaktivitäten der Erziehungswissenschaft ab. Sie akzentuieren aber Entwicklungen in der Disziplin, die über die jeweils behandelten Themenbereiche hinweg von Interesse sein können. Die Ausdifferenzierung der Erziehungswissenschaft in Subdisziplinen, die interdisziplinäre Aufgeschlossenheit der Erziehungswissenschaft, ihre Bedeutung als Referenzdisziplin pädagogischer Profession und die seismographische Sensibilität der Erziehungswissenschaft für Veränderungen im Bildungssystem sowie in der öffentlichen Diskussion über Erziehung und Bildung spiegeln sich in den Themenschwerpunkten wider.

In der Transferforschung befasst sich die Erziehungswissenschaft selbstreflexiv mit den Folgen ihrer eigenen Forschung für die Praxis und den Rückwirkungen der Praxisrelevanz auf ihre Forschungskonzeptionen. Transfer wird in diesem Kontext mit dem Stichwortartikel von Gräsel (2010, S.7) als „Verbreitung wissenschaftlich fundierter Innovationen im Bildungssystem" eingeführt. Ausgehend davon verdeutlicht Gräsel die Position der erziehungswissenschaftlichen Forschung in unterschiedlichen Transferstra-

Online publiziert: 20.04 .2011

(C) VS Verlag für Sozialwissenschaften 2011

Prof. Dr. H. Kuper $(\bowtie)$

Arbeitsbereich Weiterbildung und Bildungsmanagement, Freie Universität Berlin, Arnimallee 12,

14195 Berlin, Deutschland

E-Mail: harm.kuper@fu-berlin.de 
tegien und weist auf Variationen in den Grundmustern der Forschungsdesigns hin. Gräsel votiert für eine Konzeption, in der Forschung ,zentrales Element der Innovation“ und weniger „Begleitforschung“ ist (2010, S. 17). Forschung komme so eine strategisch wichtige Rolle im Transfer zu. Ist Transfer geheimnisvoll, wie Prenzel (2010) im Titel seines Beitrags fragt? Seine Antwort kann zusammengefasst werden: Ja, sofern das Abschleifen von Innovationen in den Routinen und der Evolution des Bildungssystems im Fokus der Betrachtung liegt. Aber das Geheimnis kann gelüftet werden, sofern die Forschung ihre Verantwortung für die Entwicklung des Innovationspotenzials, das mit wissenschaftlichem Wissen transportiert wird, offensiv wahrnimmt. Dieser Standpunkt hat - wie Prenzel zeigt - Folgen für die disziplinäre Verständigung über methodische Standards der Forschung, die Förderstruktur und die Indikatorisierung von Forschungsleistungen der Erziehungswissenschaft. Von einer Vielzahl konkreter Projekte der Transferförderung und Transferforschung legen die beiden weiteren Beiträge des Schwerpunktes Zeugnis ab: Reinhold Nickolaus, Annette Gönnenwein und Cordula Petsch (Nickolaus et al. 2010) beziehen sich auf die Modellversuchsförderung der Bund-Länder-Kommission und die auf Transfer ausgelegten Programme des Bundesministeriums für Bildung und Forschung. Neben empirischen Befunden über transferförderliche und transferhemmende Bedingungen zeigen ihre Ausführungen auch die generelle Abhängigkeit erziehungswissenschaftlicher Transferprojekte vom politischen Willen, Modellversuche in größerem Umfang zu initiieren, konzeptionell auszugestalten und administrativ zu unterstützen. Gerade weil Transfer und Transferforschung nicht ausschließlich den Funktionslogiken der Wissenschaft folgen - so lassen sich die Ausführungen von Nickolaus et al. (2010) resümieren - sind viele Fragen zu den Transfereffekten bislang unbeantwortet und geben Anlass für weitere Forschung. Eine wissenschaftstheoretisch sehr luzide und an einzelnen Projekten exemplifizierte Konzeption dazu entwickelt Einsiedler (2010): Ausgehend von Überlegungen zur Relevanz, zur Technologie und zur Kausalität, mit denen er Fragen nach der Anwendbarkeit wissenschaftlichen Wissens adressiert, legt er ein Modell der Didaktischen Entwicklungsforschung an, das neben der wissenschaftlich-methodischen auch die strategische Ausrichtung innovationsförderlicher Projekte befördern kann.

Die Zivilgesellschaft ist ein soziales Emergenzphänomen, das gleichermaßen Bedingungen individueller Entwicklung stellt, wie es selbst der individuellen Entwicklung bedarf. Die Autoren der Beiträge des zweiten ZfE-Schwerpunktes im Jahr 2010 arbeiten zivilgesellschaftliches Engagement als das erziehungswissenschaftlich relevante Forschungsthema aus diesem Zusammenhang heraus. Priller (2010) stellt in seinem Stichwortartikel die thematischen Schnittstellen der Engagementforschung mit der Erziehungswissenschaft dar und periodisiert ihren Verlauf anhand vorliegender Studien. Dabei verdeutlicht er den Bedarf an einer interdisziplinären Entwicklung theoretisch-konzeptioneller und empirischer Grundlagenforschung, in der sich die Erziehungswissenschaft noch positionieren kann. Verfolgens- bzw. vertiefenswerte Anknüpfungspunkte zeigen die beiden empirischen Beiträge des Schwerpunktes auf: Gensicke (2010) dokumentiert Befunde aus dem Freiwilligensurvey, die im Trend ein ansteigendes zivilgesellschaftliches Engagement belegen. Dabei wird freiwilliges Engagement insbesondere in jüngeren Bevölkerungsgruppen als Möglichkeit des informellen Qualifikationserwerbs genutzt. Damit ist ebenso ein erziehungswissenschaftliches Kernthema berührt, wie mit dem Befund einer besonders rapiden Steigerung des ehrenamtlichen Engagements bei älteren 
Personen, der Fragen nach dem Generationenverhältnis aufwirft. Youniss und Reinders (2010) rekapitulieren zunächst die US-amerikanische Diskussion um gemeinschafsförderliches Engagement von Jugendlichen; ihr entnehmen sie die Frage nach den Effekten unterschiedlicher Formen des Engagements auf die soziale Identität von Jugendlichen und ihr längerfristiges Engagement. Die regressionsanalytische Modellierung und empirische Überprüfung dieses Effektes jeweils anhand einer in den USA und in Deutschland gezogenen Stichprobe gibt einen Beleg für das hohe Erkenntnispotenzial kulturvergleichender erziehungswissenschaftlicher Forschung.

Die Entwicklung der erziehungswissenschaftlichen Forschung ist unter anderem von einer deutlich hervortretenden subdisziplinären Struktur und der Herausbildung diskursiver Milieus beeinflusst, in denen vielfältige Theorietraditionen bewahrt, fortgeführt und für die Erziehungswissenschaft erschlossen werden. Die theoretische Aufbereitung erziehungswissenschaftlicher Forschungsthemen kann dabei innovative Impulse über die zuständigen Subdisziplinen hinaus entfalten. Entsprechende Angebote machen die Beiträge zum dritten Schwerpunkt Gestaltungsspielräume des Lernens Erwachsener. Die organisatorisch versatilen und eng mit den strukturellen Auswirkungen des gesellschaftlichen Wandels verkoppelten Rahmenbedingungen des Lernens Erwachsener bedingen in der Erwachsenenbildungs- und Weiterbildungsforschung die Attraktivität grundlagentheoretischer sozialwissenschaftlicher Zugänge. So lassen sich die in der Diskussion um das Lernen Erwachsener eröffneten Referenzmuster systemtheoretisch fassen (Kuper 2010) und die individuelle Nutzung resp. Gestaltung von Lehr-Lern-Settings erschließt sich aus einer interaktionstheoretischen Perspektive (Wittpoth 2010). Auf diesen Grundlagen basieren in der Weiterbildungsforschung vielfach Studien mit explorativen Fragestellungen; im ZfE-Schwerpunkt sind sie repräsentiert durch eine Arbeit Dinkelakers (2010), in der Ergebnisse aus einer Mikroanalyse der Aufmerksamkeitsfokussierung von Teilnehmenden in Kursen der Erwachsenenbildung vorstellt werden, und durch eine diskursanalytischen Reflexion über Macht in erwachsenenpädagogischer Interaktion (Nolda 2010). Einen Vorläufer jüngerer Ansätze der Forschung zum Lehren und Lernen aus der Erwachsenenbildung in den 1920er-Jahren rezipiert Seitter (2010) in einem Beitrag über Alfred Manns Praktiken der Unterrichtsprotokollierung. Diese Arbeit ist in ihrer Darstellung der von Mann begründeten Verknüpfung von Forschung, didaktischer Planung und Professionalisierung wissenschaftsgeschichtlich von ebenso großer wie systematischer Bedeutung.

Sprache ist zentrales Medium der Bildung und Mehrsprachigkeit - unser viertes Schwerpunktthema - eine der zentralen Herausforderungen für die schulische Bildung. Die erziehungswissenschaftlichen Forschungsbefunde und Positionen zu diesem Thema reflektieren die durch Migration bedingten Entwicklungen, die Mehrsprachigkeit als gesellschaftliche Realität zur Folge haben; sie stehen im Spannungsfeld einer Kontroverse um die Förderung der Zwei- und Mehrsprachigkeit in der schulischen Bildung. Gogolin (2010) bilanziert in dem einführenden Überblicksbeitrag die einschlägigen Diskussionsstränge und zeigt Perspektiven für theoretische und empirische Studien zur sprachlichen Diversität auf. Eine davon vertiefen Creese und Blackledge (2010) mit dem Konzept der Super-Diversität. Sie zeigen mit den Mitteln soziolinguistischer Analyse anhand von Fallstudien die Amalgamierung von Sprachen in der schulischen Kommunikation unter mehrsprachigen Personen auf. Die weiteren Beiträge schließen von der theoriegeleiteten 
Reflexion zur Anwendung wissenschaftlichen Wissens im professionellen Handlungsfeld auf. So stellen Gantefort und Roth (2010) im Kontext des BLK-Programms FörMig entwickelte Instrumente der Sprachdiagnostik vor; Lengyel (2010) präsentiert anhand von Unterrichtsbeobachtungen Konzepte zur Förderung der Bildungssprache; der Beitrag von Holger Hopp, Dieter Thoma und Rosemarie Tracy (Hopp et al. 2010) setzt diese Ausrichtung konsequent fort, indem die Grundzüge eines Modells der Sprachförderkompetenz pädagogischer Fachkräfte entworfen werden.

Neben den Schwerpunkten vervollständigen die Beiträge im Allgemeinen Teil der ZfE auch im Jahre 2010 den Blick auf das Spektrum der erziehungswissenschaftlichen Forschung: Sie erstrecken sich von der empirischen Bildungsforschung bis zur Bildungsphilosophie, von bildungspolitisch aktuellen, steuerungsrelevanten Fragestellungen bis zur Grundlagenforschung und über eine breite Palette von Forschungsthemen aus professionalisierten pädagogischen Handlungsfeldern. Auch für diese Beiträge geben die Prämissen der ZfE - hohe fachwissenschaftliche Standards, Interdisziplinarität und Verantwortung gegenüber dem wissenschaftlichen Informationsbedarf im Bildungswesen - die Orientierung vor.

Im Namen der Herausgeberinnen und Herausgeber danke ich Ihnen - liebe Leserinnen und Leser - für Ihr Interesse an der ZfE, das gleichzeitig auch Ausdruck an einer forschungsstarken Erziehungswissenschaft ist.

Wir freuen uns auf einen Beitrag aus Ihrer Forschung für die ZfE!

Ihr

Harm Kuper

\section{Literatur}

Creese, A., \& Blackledge, A. (2010). Towards a sociolinguistics of superdiversity. Zeitschrift für Erziehungswissenschaft, 13(4), 549-572.

Dinkelaker, J. (2010). Aufmerksamkeitsbewegungen: Zur Prozessierung der Teilnahme in Kursen der Erwachsenenbildung/Weiterbildung. Zeitschrift für Erziehungswissenschaft, 13(3), $377-392$.

Einsiedler, W. (2010). Didaktische Entwicklungsforschung als Transferförderung. Zeitschrift für Erziehungswissenschaft, 13(1), 59-81.

Gantefort, C., \& Roth, H.-J. (2010). Sprachdiagnostische Grundlagen für die Förderung bildungssprachlicher Fähigkeiten. Zeitschrift für Erziehungswissenschaft, 13(4), 573-591.

Gensicke, T. (2010). Zivilgesellschaft und freiwilliges Engagement in Deutschland im Lichte des Freiwilligensurveys 1999 - 2004 - 2009. Zeitschrift für Erziehungswissenschaft, 13(2), $215-232$.

Gogolin, I. (2010). Stichwort: Mehrsprachigkeit. Zeitschrift für Erziehungswissenschaft, 13(4), $529-547$.

Gräsel, C. (2010). Stichwort: Transfer und Transferforschung im Bildungsbereich. Zeitschrift für Erziehungswissenschaft, 13(1), 7-20.

Hopp, H., Thoma, D., \& Tracy, R. (2010). Sprachförderkompetenz pädagogischer Fachkräfte: Ein sprachwissenschaftliches Modell. Zeitschrift für Erziehungswissenschaft, 13(4), 609-629. 
Kuper, H. (2010). Stichwort: Erziehungswissenschaftliche Perspektiven auf das Lernen Erwachsener. Zeitschrift für Erziehungswissenschaft, 13(3), 345-362.

Lengyel, D. (2010). Bildungssprachförderlicher Unterricht in mehrsprachigen Lernkonstellationen. Zeitschrift für Erziehungswissenschaft, 13(4), 593-608.

Nickolaus, R., Gönnenwein, A., \& Petsch, C. (2010). Die Transferproblematik im Kontext von Modellversuchen und Modellversuchsprogrammen. Zeitschrift für Erziehungswissenschaft, 13(1), 39-58.

Nolda, S. (2010). Macht in Lehr-/Lerninteraktionen Erwachsener: Machtfeststellungen in Interaktionsanalysen von Lehr-Lernsituationen der Erwachsenenbildung in Abhängigkeit von Machtkonzepten und Datentypen. Zeitschrift für Erziehungswissenschaft, 13(3), 405-419.

Prenzel, M. (2010). Geheimnisvoller Transfer? Wie Forschung der Bildungspraxis nützen kann. Zeitschrift für Erziehungswissenschaft, 13(1), 21-37.

Priller, E. (2010). Stichwort: Vom Ehrenamt zum zivilgesellschaftlichen Engagement. Zeitschrift für Erziehungswissenschaft, 13(2), 195-213.

Seitter, W. (2010). Die Arbeitsgemeinschaft als partizipative Regulationsform: Alfred Manns Unterrichtsprotokollierungen im Kontext erwachsenenpädagogischer Lehr-/Lernforschung der 1920er-Jahre. Zeitschrift für Erziehungswissenschaft, 13(3), 393-404.

Wittpoth, J. (2010). Spielräume des Selbst in Lehr-Lern-Settings Erwachsener? Zeitschrift für Erziehungswissenschaft, 13(3), 363-375.

Youniss, J., \& Reinders, H. (2010). Youth and community service: A review of U.S. research, a theoretical perspective, and implications for policy in Germany. Zeitschrift für Erziehungswissenschaft, 13(2), 233-248. 